\title{
End-of-Life Healthcare Utilization of Older Mexican Americans With and Without a Diagnosis of Alzheimer's Disease and Related Dementias
}

\author{
Christine Nguyen, BS, ${ }^{1, *}$ Brian Downer, $\mathrm{PhD},{ }^{2}$ Lin-Na Chou, $\mathrm{MS}_{,}{ }^{3}$ Yong-Fang Kuo, $\mathrm{PhD},{ }^{3}$ \\ and Mukaila Raji, MD, MS, FACP4
}

${ }^{1}$ School of Medicine, ${ }^{2}$ Division of Rehabilitation Sciences, School of Health Professions, ${ }^{3}$ Office of Biostatistics, Preventive Medicine and Community Health, and ${ }^{4}$ Division of Geriatrics, Internal Medicine, The University of Texas Medical Branch at Galveston.

*Address correspondence to: Christine Nguyen, BS, School of Medicine, The University of Texas Medical Branch at Galveston, 301 University Blvd, Galveston, TX 77555. E-mail: chynguye@utmb.edu

Received: August 13, 2018; Editorial Decision Date: February 19, 2019

Decision editor: Anne Newman, MD, MPH

\begin{abstract}
Background: Little is known about the patterns of end-of-life health care for older Mexican Americans with or without a diagnosis of Alzheimer's disease and related dementias (ADRD). Our objective was to investigate the frequency of acute hospital admissions, intensive care unit use, and ventilator use during the last 30 days of life for deceased older Mexican American Medicare beneficiaries with and without an ADRD diagnosis.

Methods: We used Medicare claims data linked with survey information from 1,090 participants (mean age of death 85.1 years) of the Hispanic Established Populations for the Epidemiologic Studies of the Elderly. Multivariable logistic regression models were used to estimate the odds for hospitalization, intensive care unit use, and ventilator use in the last 30 days of life for decedents with ADRD than those without ADRD. Generalized linear models were used to estimate the risk ratio (RR) for length of stay in hospital.

Results: Within the last 30 days of life, $64.5 \%$ decedents had an acute hospitalization $(59.1 \%$ ADRD, $68.3 \%$ no ADRD), $33.9 \%$ had an intensive care unit stay (31.3\% ADRD, 35.8\% no ADRD), and $17.2 \%$ used a ventilator (14.9\% ADRD, $18.8 \%$ no ADRD). ADRD was associated with significantly lower hospitalizations (odds ratio $[\mathrm{OR}]=0.67,95 \%$ confidence interval $[\mathrm{CI}]=0.50-0.89$ ) and shorter length of stay in hospital $(\mathrm{RR}=0.77,95 \% \mathrm{CI}=0.65-0.90)$.

Conclusion: Hospitalization, intensive care unit stay, and ventilator use are common at the end of life for older Mexican Americans. The lower hospitalization and shorter length of stay in hospital of decedents with ADRD indicate a modest reduction in acute care use. Future research should investigate the impact of end-of-life planning on acute-care use and quality of life in terminally ill Mexican American older adults.
\end{abstract}

Keywords: Medicare, Mexican Americans, Terminal care, Minority aging, Health services.

Many older adults experience fragmented care across a myriad of health care settings in the months before death $(1,2)$. From 2000 to 2009 , the average number of health care transitions during the 90 days before death increased from 2.1 to 3.1 transitions, with the percentage of patients experiencing a health care transition within 3 days of death increasing from $10.3 \%$ to $14.2 \%$ (1). However, this percentage decreased to $11.2 \%$ in 2011 and $10.8 \%$ in 2015 (3). The percentage of patients who experienced three or more hospitalizations in the 90 days before death has also decreased from $11.5 \%$ in 2009 to $7.1 \%$ in 2015 (3).
Prior research on end-of-life (EOL) care for older adults has generally focused on non-Hispanic white older adults and few studies have used data that include minority populations, especially older Hispanics. Hispanics are the largest minority population in the United States with the majority of Hispanics being of Mexican descent (4). Hispanics' decision making regarding EOL care is shaped by cultural values and beliefs. Family has a critical role in EOL care decision making (5) and older Hispanics may prefer less autonomy in EOL care decision making $(5,6)$. Although not reported in all studies $(5,7)$, evidence supports that older Hispanics prefer to receive 
life-sustaining treatments $(8,9)$ and more aggressive EOL care $(10$ 12). These preferences may reflect cultural beliefs that aggressive treatment can lead to recovery (9) and that a physician would not suggest aggressive treatment in situations where there was no chance for recovery (8).

Alzheimer's disease is the sixth leading cause of death in the United States (13). Approximately one-third of Medicare beneficiaries who died in 2014 had been diagnosed with Alzheimer's disease and related dementias (ADRD) (14). This percentage may be even higher for Hispanic Medicare beneficiaries as the prevalence of ADRD is higher among Hispanics than non-Hispanic whites (15).

ADRD is an increasingly common cause of death (16), but few studies have investigated differences in EOL care between older adults with and without ADRD $(1,17)$. The available evidence is mixed with ADRD decedents having fewer inpatient and outpatient visits but greater use of skilled nursing and hospice services during the last 6 months of life than decedents without ADRD (17). There is also evidence for decreasing trends in the percentage of ADRD decedents who are hospitalized during the last 90 days of life, but increasing trends for nursing home stays in the last 90 days of life and intensive care unit (ICU) use in the last 30 days of life (1).

Little data exist on the patterns and frequency of EOL health care utilization for older Mexican Americans, especially for those who have been diagnosed with ADRD. We used Medicare claims data linked with survey information to determine the frequency of and variations in acute hospital admissions, ICU use, and ventilator use during the last 30-days of life in deceased older Mexican American Medicare beneficiaries with and without an ADRD diagnosis.

\section{Methods}

\section{Sample Population}

This analysis used data from the Hispanic Established Populations for the Epidemiologic Studies of the Elderly (H-EPESE) that has been linked with Medicare claims data files. The H-EPESE is an ongoing, longitudinal study of Mexican Americans aged 65 and older living in the southwestern United States (18). A total of 3,050 participants were enrolled at the first observation wave in 1993-1994 and nine observation waves have been completed as of 2016. At wave 5 (2004/2005), a new cohort of 902 participants aged 75 and older was added to the sample.

The H-EPESE survey files have been linked with Medicare Master Beneficiary Summary Files, Medicare Provider Analysis and Review files, Outpatient Standard Analytic files, and Carrier files for 19992012. The linked data were supplied by the Centers for Medicare and Medicaid Services following the procedures in a data use agreement approved by Centers for Medicare and Medicaid Services and our University's Institutional Review Board. The data linkage was done by sending the social security numbers for H-EPESE participants alive after December 31, 1998 to the Centers for Medicare and Medicaid Services $(n=3,348)$. The records for participants who could not be matched according to social security numbers and gender or who did not have a unique ID for both the H-EPESE survey data set and Medicare claims data were not considered to be a successful match. The records for 2,585 H-EPESE participants of a possible 3,348 participants were successfully matched. With the exception of age, there were no significant differences for gender, nativity, language of interview, or education between the 2,585 participants who were successfully matched and the 3,348 participants who were alive after December 31, 1998 (Supplementary Table 1).
Twenty-eight percent participants who were successfully matched were aged 80 years or older in 1999 compared to $26.1 \%$ of the 3,348 participants who were alive after December 31, 1998.

A summary of the sample selection is presented in Figure 1. The final sample $(n=1,090)$ was selected from 2,585 H-EPESE participants who were linked with Medicare claims files. Participants who were recorded as deceased in the H-EPESE as determined by the National Death Index and Medicare claims data were selected ( $n=1,778)$. We excluded 102 participants whose date of death in the National Death Index did not match with the date of death in the Medicare claims files $(n=1,676)$. We excluded 523 participants who were not continuously enrolled in Medicare during the 13 months before death so that we had a look back period to identify ADRD and other health conditions in the Medicare claims data ( $n=1,153)$. The sample of 1,676 participants was slightly but significantly older than the sample of 1,153 participants who were continuously enrolled in Medicare during the 13 months before death (Supplementary Table 1). We excluded an additional 63 participants who did not have valid information to determine ADRD status (see Classification of ADRD section). The regression models used data from 1,001 participants who did not have any missing data for sociodemographic characteristics.

Medicare claims data do not include information on a person's daily functioning, cognition, and depressive symptoms. These measures are routinely collected in population-based cohort studies and may affect EOL care. We conducted secondary analyses to examine differences in EOL care according to limitations in activities of daily living (ADL), impaired cognitive functioning, and high depressive symptoms. These analyses used data from participants whose last survey interview was completed within 2 years of death and who had continuous Medicare enrollment between the last survey interview

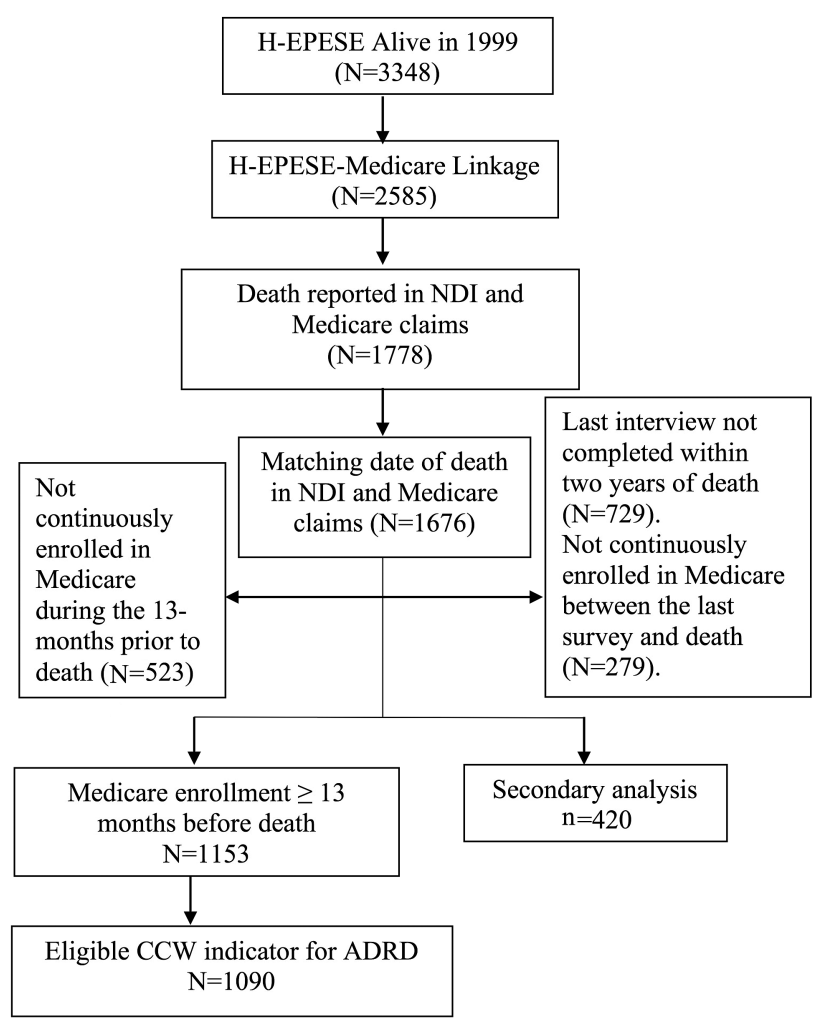

Figure 1. Flowchart of selecting study subjects. 
and death. We excluded 248 participants whose last interview had to be completed by a proxy or had missing data for sociodemographic characteristics, cognitive functioning, ADL limitations, or depressive symptoms. The final sample for the secondary analyses included 420 participants.

\section{Classification of ADRD}

We used the Chronic Conditions Data Warehouse indicator variable to identify participants with ADRD $(19,20)$. The Chronic Conditions Data Warehouse includes variables for 27 common health conditions. These variables are created using algorithms of International Classification of Diseases, Ninth Revision (ICD-9) diagnostic codes that are applied to administrative claims data (21). These variables are available in the Medicare Master Beneficiary Summary Files. The Chronic Conditions Data Warehouse algorithm for ADRD or senile dementia includes 24 ICD-9 codes. Medicare beneficiaries who had one or more of the 24 ICD-9 codes in at least one inpatient, skilled nursing facility, home health, outpatient, or carrier file were identified as having a diagnosis of ADRD (20).

\section{EOL Care Measures}

The EOL care measures included acute hospitalization, ICU use, and ventilator use. These outcomes were each treated as dichotomous variables (yes or no). Length of stay (LOS) for participants who did not experience a hospitalization was recorded as zero days. All outcome measures were determined using the Medicare Provider Analysis and Review file. Acute hospitalizations were identified according to if a participant had a discharge date that was within 30 days of death. The Medicare Provider Analysis and Review file includes indicator codes for if a participant received care in the ICU. Finally, ventilator use was determined by four ICD-9 procedure codes (93.90, 96.70, 96.71, and 96.72).

\section{Covariates}

We selected covariates based on prior EOL care studies and potential confounders relevant to EOL care among older Mexican Americans. These included participants' demographic characteristics and comorbid health conditions. The demographic characteristics were selected from the H-EPESE survey and included age at death (68-79, 80-84, 85-89, and 90+), gender, country of birth (US-born or foreign-born), and years of formal education. The categories for age at death were selected so that the sample sizes in each category were relatively equal. Also, the youngest age at death was 68 years. Health conditions that were controlled for in the analysis included diabetes, heart failure, stroke, chronic obstructive pulmonary disease, and cancer (colorectal, endometrial, female breast, lung, and prostate). These conditions were identified using the chronic condition categories in the Medicare Chronic Conditions Data Warehouse and were defined as having a recorded fee-for-service claim during the last year of life. The Charlson Comorbidity Index (CCI) score was also calculated to examine differences in EOL care according to the number of health comorbidities. The CCI score was calculated using inpatient and outpatient claims files from the 13 months before death, excluding the last month of life. The CCI score was used to group patients into four categories $(0,1-2,3-4$, and 5+).

The secondary analyses included as covariates the demographic characteristics from the primary analyses, as well as marital status (married, widowed, other), living alone (yes or no), self-reported health conditions (diabetes, heart attack, heart failure, stroke, chronic obstructive pulmonary disease, cancer, and fracture), limitations in one or more ADLs, cognitive impairment, and depression. ADL limitations were determined by asking participants if at the time of the interview they needed help from another person or special equipment to walk across a small room, bath, personal grooming, get dressed, eat, move from a bed to a chair, or use the toilet. Cognitive impairment was defined as a score of less than 18 points Mini-Mental Status Exam (22). Depression was defined as scoring at least16 points on the Center for Epidemiologic Studies Depression Scale (23).

\section{Statistical Analysis}

Descriptive statistics were presented as percentage and mean (standard deviation). Chi-square tests and analysis of variance were used to make group comparisons in sociodemographic and health characteristics according to acute hospitalization, ICU use, and ventilator use. Multivariable logistic regression models were used to estimate the odds ratio (OR) for hospitalization, ICU use, and ventilator use in the last 30 days of life for decedents with ADRD than those without ADRD, controlling for demographic and health characteristics. Generalized linear models with log-link normal distribution were used to estimate the risk ratio (RR) for the LOS. All analyses were conducted using SAS statistical software (version 9.4; SAS Institute Inc., Cary, NC).

\section{Results}

\section{Sample Description}

Approximately $65 \%$ decedents had an acute hospitalization, $33.9 \%$ had an ICU stay, and $17.2 \%$ used a ventilator in the last 30 days of life (Table 1). The average LOS was 6.0 days (median: 4.0 days). Decedent characteristics associated with greater EOL care included younger age at death, being born in Mexico, interviewed in Spanish, diabetes, heart failure, and chronic obstructive pulmonary disease (Table 1$)$.

\section{Differences in EOL Care by ADRD Diagnosis}

A total of 450 decedents $(41.3 \%)$ had a diagnosis of ADRD. Decedents diagnosed with ADRD had significantly lower odds for hospitalization $(\mathrm{OR}=0.67,95 \% \mathrm{CI}=0.50-0.89)$ and 0.77 times shorter LOS (95\% CI = 0.65-0.90; Table 2). Decedents with ADRD also had lower odds to have had an ICU stay $(\mathrm{OR}=0.76,95 \%$ $\mathrm{CI}=0.56-1.02)$ and to have used a ventilator $(\mathrm{OR}=0.70,95 \%$ $\mathrm{CI}=0.48-1.02)$, but these findings only approached statistical significance.

Other measures associated with EOL care included younger age at death, place of birth, diabetes, heart failure, and stroke (Table 2). A significant decreasing trend in the odds for hospitalization, ICU use, and ventilator use with advancing age at death was observed (all trend tests $p<.01$ ). Compared to decedents who died between 65 and 79 years of age, those who were 90 years or older had significantly lower odds to have been hospitalized $(\mathrm{OR}=0.62,95 \% \mathrm{CI}=0.39-$ 0.97), to have stayed in the $\mathrm{ICU}(\mathrm{OR}=0.61,95 \% \mathrm{CI}=0.39-0.95)$, or to have used a ventilator $(\mathrm{OR}=0.49,95 \% \mathrm{CI}=0.28-0.86)$ in the last 30 days of life. In addition, patients who died between 85 and 89 years of age had significantly lower odds to have used a ventilator $(\mathrm{OR}=0.49,95 \% \mathrm{CI}=0.28-0.84)$. Decedents born in the United States had $0.65(95 \% \mathrm{CI}=0.49-0.86)$ times lower odds to have an ICU stay compared to foreign-born decedents, but the differences in hospitalization and ventilator use according to nativity were not statistically significant. Heart failure was associated with $1.43(95 \%$ 
Table 1. Baseline Characteristics and Percent of Patients Experiencing End-of-Life Care Measures in Medicare Cohort $(N=1,090)$

\begin{tabular}{|c|c|c|c|c|c|}
\hline \multirow[b]{2}{*}{ Characteristics" $†$} & \multirow[b]{2}{*}{$N$} & \multicolumn{3}{|c|}{ End-of-life care measure, \% Yes } & \multirow{2}{*}{$\frac{\text { Mean }(S D)}{\operatorname{LOS}(\mathrm{d})}$} \\
\hline & & Hospitalization & ICU & Ventilation & \\
\hline Total & 1,090 & 64.5 & 33.9 & 17.2 & $6.0(7.35)$ \\
\hline \multicolumn{6}{|l|}{ Age at death } \\
\hline $68-79 y$ & 201 & 72.6 & 38.3 & 21.9 & $7.0(7.68)$ \\
\hline $80-84$ y & 318 & 66.4 & 38.7 & 20.8 & $6.8(8.29)$ \\
\hline $85-89 y$ & 282 & 61.3 & 30.5 & 14.2 & $5.5(6.91)$ \\
\hline $90+y$ & 289 & 59.9 & 29.1 & 12.8 & $4.8(6.18)$ \\
\hline \multicolumn{6}{|l|}{ Gender } \\
\hline Male & 458 & 64.6 & 36.7 & 19.2 & $6.1(7.78)$ \\
\hline Female & 632 & 64.4 & 32.0 & 15.7 & $5.9(7.03)$ \\
\hline \multicolumn{6}{|l|}{ Place of birth } \\
\hline Mexico & 439 & 65.8 & 37.8 & 17.8 & $6.0(7.41)$ \\
\hline United States & 651 & 63.6 & 31.3 & 16.7 & $5.9(7.32)$ \\
\hline \multicolumn{6}{|l|}{ Language } \\
\hline English & 216 & 61.1 & 26.9 & 13.0 & $5.3(6.94)$ \\
\hline Spanish & 874 & 65.3 & 35.7 & 18.2 & $6.1(7.44)$ \\
\hline \multicolumn{6}{|l|}{ Education $^{\mathrm{c}}$} \\
\hline$<12 \mathrm{y}$ & 904 & 65.7 & 34.1 & 16.7 & $6.1(7.43)$ \\
\hline$\geq 12 y$ & 97 & 56.7 & 39.2 & 20.6 & $5.4(7.44)$ \\
\hline \multicolumn{6}{|l|}{ Dementia } \\
\hline No & 640 & 68.3 & 35.8 & 18.8 & $6.6(7.87)$ \\
\hline Yes & 450 & 59.1 & 31.3 & 14.9 & $5.1(6.45)$ \\
\hline \multicolumn{6}{|l|}{ Diabetes } \\
\hline No & 526 & 60.8 & 28.5 & 13.7 & $5.2(6.96)$ \\
\hline Yes & 564 & 67.9 & 39.0 & 20.4 & $6.7(7.62)$ \\
\hline \multicolumn{6}{|l|}{ Heart failure } \\
\hline No & 531 & 59.9 & 29.0 & 15.3 & $4.9(6.93)$ \\
\hline Yes & 559 & 68.9 & 38.6 & 19.0 & $7.0(7.60)$ \\
\hline \multicolumn{6}{|l|}{ Stroke } \\
\hline No & 953 & 63.5 & 33.7 & 17.2 & $5.8(7.26)$ \\
\hline Yes & 137 & 71.5 & 35.8 & 16.8 & $7.5(7.79)$ \\
\hline \multicolumn{6}{|l|}{ COPD } \\
\hline No & 790 & 62.4 & 32.9 & 16.5 & $5.6(7.11)$ \\
\hline Yes & 300 & 70.0 & 36.7 & 19.0 & $7.0(7.87)$ \\
\hline \multicolumn{6}{|l|}{ Cancer } \\
\hline No & 983 & 64.7 & 34.5 & 17.7 & $6.0(7.35)$ \\
\hline Yes & 107 & 62.6 & 29.0 & 12.1 & $5.9(7.33)$ \\
\hline
\end{tabular}

Note: $\mathrm{COPD}=$ chronic obstructive pulmonary disease; $\mathrm{ICU}=$ intensive care unit; LOS = length of stay in hospital.

"Survey information was based on the latest interview before death $†$ Missing data for education

Bold $p<.05$.

$\mathrm{CI}=1.07-1.90)$ times higher odds to be hospitalized and $1.59(95 \%$

$\mathrm{CI}=1.19-2.13)$ times higher odds to have stayed in the ICU during the last 30 days of life. Heart disease and stroke were associated with $1.37(95 \% \mathrm{CI}=1.16-1.62)$ and $1.27(95 \% \mathrm{CI}=1.06-1.52)$ times longer LOS, respectively.

A significant increasing trend in the odds to have been hospitalized in the last 30 days of life according to greater CCI score was detected (Table 3). Compared to decedents with a CCI score of 0 , those with a CCI score of $1-2,3-4$, and $5+$ had 2.14 (95\% CI=1.20-3.80), 2.82 (95\% CI=1.57-5.06), and 3.27 (95\% CI=1.92-5.57) higher odds, respectively, to have been hospitalized in the 30 days before death (trend test $p<.05$ ).

The results for the secondary analyses are presented in Table 4. No significant differences in the EOL care measures between patients with one or more ADL limitations or depression were detected. Patients who were classified as cognitively impaired had 0.55 times lower odds (95\% $\mathrm{CI}=0.33-0.91)$ to have stayed in the ICU. The odds to have been hospitalized were not significantly different according to cognitive status.

\section{Discussion}

This study aimed to describe the EOL care for older Mexican Americans by identifying acute hospitalizations, ICU use, and ventilation use in the last 30 days of life. We observed that $65 \%$ decedents were hospitalized, $33.9 \%$ stayed in the ICU, and $17.2 \%$ used a ventilator in the last 30 days of life. These percentages are higher than what has been reported by other studies using data from predominately non-Hispanic white populations $(1,3)$. The greater health care utilization at the end of life in our sample may reflect ethnic differences in advanced care planning. Advanced care planning has been associated with fewer hospitalizations (24) and less stress, anxiety, and depression among family members (25). Hispanics are less likely than non-Hispanic whites to complete an advanced directive and to have discussions with family members and health care professionals about the type of care they want to receive at the (26). This disparity may be due to several factors including a lack of awareness about advanced care resources (26), language barriers and low 
Table 2. Multiple Logistic Regression Model for the Association Between ADRD and End-of-Life Care $(N=1,001)$

\begin{tabular}{|c|c|c|c|c|}
\hline \multirow[b]{2}{*}{ Characteristics } & \multicolumn{3}{|c|}{ End-of-life care measure, OR (95\% CI) } & \multirow{2}{*}{$\frac{\text { Length of stay }}{\text { Ratio }(95 \% \mathrm{CI})}$} \\
\hline & Hospitalization & ICU & Ventilation & \\
\hline ADRD & $0.67(0.50-0.89) *$ & $0.76(0.56-1.02)$ & $0.70(0.48-1.02)$ & $0.77(0.65-0.90)^{*}$ \\
\hline \multicolumn{5}{|c|}{ Age at death (ref: $68-79$ y) } \\
\hline $80-84$ y & $0.83(0.55-1.26)$ & $0.97(0.65-1.44)$ & $0.92(0.57-1.47)$ & $0.99(0.83-1.19)$ \\
\hline $85-89 y$ & $0.71(0.46-1.09)$ & $0.69(0.45-1.05)$ & $0.49(0.28-0.84)^{*}$ & $0.81(0.66-1.01)$ \\
\hline $90+y$ & $0.62(0.39-0.97) *$ & $0.61(0.39-0.95)^{*}$ & $0.49(0.28-0.86)^{*}$ & $0.72(0.56-0.91)^{*}$ \\
\hline Female & $1.14(0.86-1.51)$ & $0.87(0.65-1.15)$ & $0.83(0.58-1.17)$ & $1.03(0.88-1.19)$ \\
\hline US-born & $0.87(0.66-1.16)$ & $0.65(0.49-0.86)^{*}$ & $0.76(0.53-1.07)$ & $0.95(0.82-1.10)$ \\
\hline$\geq 12 \mathrm{y}$ & $0.71(0.45-1.11)$ & $1.35(0.86-2.14)$ & $1.39(0.80-2.42)$ & $0.93(0.72-1.21)$ \\
\hline Diabetes & $1.28(0.97-1.70)$ & $1.37(1.03-1.82)^{*}$ & $1.45(1.01-2.09)^{*}$ & $1.12(0.96-1.31)$ \\
\hline Heart failure & $1.43(1.07-1.90)^{*}$ & $1.59(1.19-2.13)^{*}$ & $1.28(0.89-1.85)$ & $1.37(1.16,1.62)^{*}$ \\
\hline Stroke & $1.40(0.91-2.16)$ & $0.99(0.65-1.49)$ & $0.89(0.53-1.50)$ & $1.27(1.06-1.52)^{*}$ \\
\hline COPD & $1.26(0.91-1.74)$ & $1.03(0.75-1.41)$ & $1.16(0.79-1.71)$ & $1.15(0.99-1.35)$ \\
\hline Cancer & $0.85(0.54-1.34)$ & $0.68(0.43-1.09)$ & $0.56(0.30-1.04)$ & $0.88(0.68-1.13)$ \\
\hline
\end{tabular}

Note: $\mathrm{ADRD}=$ Alzheimer's disease and related dementias; $\mathrm{CI}=$ confidence interval; $\mathrm{COPD}=$ chronic obstructive pulmonary disease; ICU = intensive care unit; OR = odds ratio.

" $p<.05$.

Table 3. Multivariable Regression Models for the Association Between Charlson Comorbidity Score and EOL $(N=1,001)$

\begin{tabular}{llllll}
\hline & \multicolumn{2}{l}{ End-of-life care measure, OR $(95 \% \mathrm{CI})$} & & \multirow{2}{*}{ Length of stay } \\
\cline { 2 - 4 } Characteristics & Hospitalization & ICU & Ventilation & & Ratio $(95 \%$ CI $)$ \\
\hline Charlson Comorbidity Score (ref: 0$)$ & $2.14(1.20-3.80)^{*}$ & $1.66(0.86-3.20)$ & $1.11(0.50-2.47)$ & $1.85(0.82-4.17)$ \\
$1-2$ & $2.82(1.57-5.06)^{*}$ & $1.76(0.91-3.39)$ & $1.36(0.62-2.97)$ & $2.46(1.11-5.46)$ \\
$3-4$ & $3.27(1.92-5.57)^{*}$ & $1.93(1.05-3.53)^{*}$ & $1.22(0.59-2.51)$ & $3.57(1.64-7.79)$ \\
$5+$ & $<0.001$ & 0.0561 & 0.6157 & $<0.001$ \\
Trend test & & & & \\
\hline
\end{tabular}

Note: $\mathrm{CI}=$ confidence interval; $\mathrm{EOL}=$ end of life; ICU = intensive care unit; OR = odds ratio. Models controlled for year of death, age at death, gender, nativity, and education.

$* p<.05$.

health literacy $(7,27)$, disagreement about prognosis and care goals between family members or with clinicians (28), and distrust in the medical system because of negative past experiences (29). The critical role of family in EOL care decision making among Hispanics suggests there is a need for more culturally competent health policies and patient-centered education that facilitate patient and family decision making (5). Implementing the use of Physician Orders for Life-Sustaining Treatment documents for patients, with the involvement of family being an option, is another possibility to ensure that EOL care is consistent with a person's values and beliefs (30).

Our findings contribute to the existing literature showing less health care utilization at the end of life among older adults with ADRD (17). We observed decedents who had been diagnosed with ADRD had significantly lower odds to have been hospitalized and had significantly shorter LOS than those who had not been diagnosed with ADRD. The lower odds for hospitalization and shorter LOS may reflect less aggressive treatment of older adults with ADRD (31). Cultural factors may also have an important role in the EOL care of older Hispanics with ADRD. Hispanic culture emphasizes the role of family in providing care for an older family member and patients may prefer to rely on other family members to make decisions on their behalf (32). Regardless, the rates of hospitalizations were still relatively high for both patient groups, with about $59 \%$ of decedents with ADRD and $68 \%$ of non-ADRD decedents spending almost a week in the hospital in the last 30 days of their life.
A strength of our analysis is we used Medicare claims files that are linked with survey data. We observed in the primary analysis that US-born Mexican Americans had significantly lower odds to stay in the ICU and to use a ventilator during the last 30 days of life compared to Mexican Americans born in Mexico. These nativity differences may reflect greater acculturation to the United States for US-born Mexican Americans (33), which could influence preferences for EOL care (29). We also examined health and functional characteristics that are not included in Medicare claims data. We observed that cognitive impairment was associated with significantly lower odds of an ICU stay, but ADL limitations and depression were not significantly associated with any EOL care measures.

However, the findings from the secondary analyses need to be interpreted with caution. Participants in the secondary analysis needed to have completed their Wave 5 interview within 2 years of death but were able to complete the interview without help from a proxy. This is a unique subsample because participants were healthy enough to have completed the interview but then died within a relatively short period of time. Our findings need to be replicated before definitive conclusions can be made about the associations between cognitive impairment, depression, ADL limitations, and EOL care.

Our study has important limitations. First, we did not have sufficient data to determine hospice, home health, inpatient, outpatient, or physician service use. There may be benefit in looking at these additional health care measures in Mexican Americans and potential 
Table 4. Multivariable Models for the Association Between ADL Limitations, Cognitive Impairment, Depression and EOL Care $(N=420)$

\begin{tabular}{llllll}
\hline & \multicolumn{2}{l}{ End of life care measure, OR $(95 \% \mathrm{CI})$} & & \multicolumn{2}{c}{ Length of stay } \\
\cline { 2 - 5 } \cline { 5 - 6 } & Hospitalization & ICU & Ventilation & Ratio $(95 \%$ CI $)$ \\
\hline Help with $\geq 1$ ADLs & $1.13(0.68-1.88)$ & $1.39(0.85-2.28)$ & $0.99(0.54-1.81)$ & $1.22(0.95-1.56)$ \\
Cognitive impairment & $0.75(0.46-1.24)$ & $0.55(0.33-0.91)^{* *}$ & $0.73(0.39-1.36)$ & $0.77(0.59-1.02)$ \\
Depression & $0.83(0.49-1.40)$ & $0.96(0.57-1.61)$ & $1.06(0.55-2.03)$ & $1.11(0.86-1.44)$ \\
\hline
\end{tabular}

Note: $\mathrm{ADL}$ = activities of daily living; $\mathrm{CI}$ = confidence interval; $\mathrm{EOL}$ = end of life; $\mathrm{ICU}$ = intensive care unit; $\mathrm{OR}$ = odds ratio. Models controlled for year of death, age at death, gender, nativity, language of interview, education, marital status, living alone, and self-reported diabetes, heart failure, stroke, and cancer.

$* p<.05$.

differences by ADRD diagnosis (17). Hospice has an important role in providing high-quality EOL care to older adults with ADRD (34) and older adults who receive hospice care experience less intense EOL care (35). Second, the H-EPESE only includes Mexican American participants and our findings may not be generalizable to other Hispanic populations living in the United States $(29,32)$. Third, our sample was limited to Medicare fee-for-service beneficiaries, and we did not have data for beneficiaries who were enrolled in Medicare Advantage plans. Recent evidence suggests decedents with Medicare Advantage may receive better quality EOL care than Medicare feefor-service beneficiaries (3). Medicare claims data also have poor sensitivity for detecting older adults who are in the early stages of ADRD (19), and it is likely that the ADRD decedents in our sample include mostly individuals in the moderate-to-severe stages of the disease. Finally, we did not have information on if decedents had an advanced directive or other documents indicating their preferences for EOL care. Advanced care planning has positive outcomes such as greater family satisfaction, better alignment with patient wishes, fewer unnecessary hospital admissions, and lower health care costs in nursing homes (36).

\section{Conclusion}

Hospitalization, ICU stay, and ventilator use are common at the end of life for older Mexican Americans. The lower hospitalization and short LOS of Mexican American decedents with ADRD likely indicate a modest attempt at de-intensification of acute care in this population. Future research is needed to investigate how EOL planning might differ for elderly Hispanics with ADRD than those who do not have ADRD. This may include investigating the role of advanced directives and Physician Orders for Life-Sustaining Treatment document use and their relationships with acute health care use and quality of life in the last 30 days of life of Hispanic elders.

\section{Supplementary Material}

Supplementary data are available at The Journals of Gerontology, Series A: Biological Sciences and Medical Sciences online.

\section{Funding}

This work was supported by the National Institute on Aging (grant numbers T35AG038048-09; R01AG010939-24) and the National Institute on Minority Health and Health Disparities (grant number R01MD010355-03).

\section{Conflict of Interest}

The authors declare no conflicts of interests.

\section{References}

1. Teno JM, Gozalo PL, Bynum JP, et al. Change in end-of-life care for Medicare beneficiaries: site of death, place of care, and health care transitions in 2000, 2005, and 2009. JAMA. 2013;309:470-477. https://www. ncbi.nlm.nih.gov/pubmed/23385273. doi: 10.1001/jama.2012.207624

2. Gozalo P, Teno JM, Mitchell SL, et al. End-of-life transitions among nursing home residents with cognitive issues. N Engl J Med. 2011;365:12121221. https://www.ncbi.nlm.nih.gov/pubmed/21991894. doi: 10.1056/ NEJMsa1100347

3. Teno JM, Gozalo P, Trivedi AN, et al. Site of death, place of care, and health care transitions among US medicare beneficiaries, 2000-2015. JAMA. 2018;320:264-271. https://www.ncbi.nlm.nih.gov/pubmed/29946682. doi: 10.1001/jama.2018.8981

4. Ortman JM, Velkoff VA, Hogan H. An Aging Nation: The Older Population in the United States. Washington, DC: U.S. Census Bureau; 2014.

5. Kelley AS, Wenger NS, Sarkisian CA. Opiniones: end-of-life care preferences and planning of older Latinos. J Am Geriatr Soc. 2010;58:1109_ 1116. https://www.ncbi.nlm.nih.gov/pubmed/20487080. doi: 10.1111/j.1532-5415.2010.02853.x

6. Savage B, Foli KJ, Edwards NE, Abrahamson K. Familism and health care provision to Hispanic older adults. J Gerontol Nurs. 2016;42:21-9; quiz 30. doi: 10.3928/00989134-20151124-03

7. Born W, Greiner KA, Sylvia E, Butler J, Ahluwalia JS. Knowledge, attitudes, and beliefs about end-of-life care among inner-city African Americans and Latinos. J Palliat Med. 2004;7:247-256. doi: $10.1089 / 109662104773709369$

8. Blackhall LJ, Frank G, Murphy ST, Michel V, Palmer JM, Azen SP. Ethnicity and attitudes towards life sustaining technology. Soc Sci Med. 1999;48:1779-1789. https://doi.org/10.1016/ S0277-9536(99)00077-5

9. Barnato AE, Anthony DL, Skinner J, Gallagher PM, Fisher ES. Racial and ethnic differences in preferences for end-of-life treatment. J Gen Intern Med. 2009;24:695-701. doi: 10.1007/s11606-009-0952-6

10. Degenholtz HB, Arnold RA, Meisel A, Lave JR. Persistence of racial disparities in advance care plan documents among nursing home residents. J Am Geriatr Soc. 2002;50:378-381. https://doi. org/10.1046/j.1532-5415.2002.50073.x.

11. Duffy SA, Jackson FC, Schim SM, Ronis DL, Fowler KE. Racial/ ethnic preferences, sex preferences, and perceived discrimination related to end-of-life care. J Am Geriatr Soc. 2006;54:150-157. doi: 10.1111/j.1532-5415.2005.00526.x

12. Ko E, Cho S, Bonilla M. Attitudes toward life-sustaining treatment: the role of race/ethnicity. Geriatr Nurs. 2012;33:341-349. doi: 10.1016/j. gerinurse.2012.01.009

13. Xu J, Murphy SL, Kochanek KD, Bastian B, Arias E. Deaths: final data for 2016. Natl Vital Stat Rep. 2018;67:1-76. https://www.ncbi.nlm.nih.gov/ pubmed/30248015.

14. Alzheimer's Association. 2017 Alzheimer's disease facts and figures. Alzheimer's and Dementia. 2017;13:325-373. doi: 10.1016/j. jalz.2017.02.001

15. Langa KM, Larson EB, Crimmins EM, Faul JD, Levine DA, Kabeto MU, et al. A comparison of the prevalence of dementia in the United States in 
2000 and 2012. JAMA Intern Med. 2017;177:51-58. https://www.ncbi. nlm.nih.gov/pubmed/27893041. doi: 10.1001/jamainternmed.2016.6807

16. Taylor CA, Greenlund SF, McGuire LC, Lu H, Croft JB. Deaths from Alzheimer's Disease-United States, 1999-2014. MMWR Morb Mortal Wkly Rep. 2017;66:521-526. https://www.ncbi.nlm.nih.gov/pub$\mathrm{med} / 28542120$. doi: $10.15585 / \mathrm{mmwr} . \mathrm{mm} 6620 \mathrm{a} 1$

17. Crouch E, Probst JC, Bennett K, Eberth JM. Differences in Medicare utilization and expenditures in the last six months of life among patients with and without Alzheimer's Disease and Related Disorders. J Palliat Med. 2019;22:126-131. doi: 10.1089/jpm.2018.0147

18. Markides KS, Stroup-Benham CA, Goodwin JS, Perkowski LC, Lichtenstein M, Ray LA. The effect of medical conditions on the functional limitations of Mexican-American elderly. Ann Epidemiol. 1996;6:386-391. https://doi.org/10.1016/S1047-2797(96)00061-0.

19. Taylor DH Jr, Fillenbaum GG, Ezell ME. The accuracy of Medicare claims data in identifying Alzheimer's disease. J Clin Epidemiol. 2002;55:929-937. https://doi.org/10.1016/S0895-4356(02)00452-3.

20. CMS Chronic Conditions Data Warehouse. CCW chronic conditions for Alzheimer's disease, related disorders, or senile dementia. 2017.

21. Chronic Conditions Data Warehouse. Retrieved November 9, 2018 https://www.ccwdata.org/

22. Folstein MF, Folstein SE, McHugh PR. "Mini-mental state". A practical method for grading the cognitive state of patients for the clinician.J Psychiatr Res. 1975;12:189-198. https://www.ncbi.nlm.nih.gov/pubmed/1202204.

23. Radloff LS. The CES-D scale: a self-report depression scale for research in the general population. Appl Psychol Meas. 1977;1:385-401. doi: 10.1177/014662167700100306

24. Molloy DW, Guyatt GH, Russo R, et al. Systematic implementation of an advance directive program in nursing homes: a randomized controlled trial. JAMA. 2000;283:1437-1444.

25. Detering KM, Hancock AD, Reade MC, Silvester W. The impact of advance care planning on end of life care in elderly patients: randomised controlled trial. BMJ. 2010;340:c1345. doi: 10.1136/bmj.c1345

26. McAfee CA, Jordan TR, Sheu JJ, Dake JA, Kopp Miller BA. Predicting racial and ethnic disparities in advance care planning using the integrated behavioral model.Omega (Westport). 2017:30222817691286.https://www. ncbi.nlm.nih.gov/pubmed/28142319. doi: 10.1177/0030222817691286

27. Barwise A, Jaramillo C, Novotny P, et al. Differences in code status and end-of-life decision making in patients with limited English proficiency in the intensive care unit. Mayo Clinic proceedings Mayo Clinic. 2018;93:1271-1281. https:/www.ncbi.nlm.nih.gov/pubmed/30100192. doi: 10.1016/j.mayocp.2018.04.021

28. Muni S, Engelberg RA, Treece PD, Dotolo D, Curtis JR. The influence of race/ethnicity and socioeconomic status on end-of-life care in the ICU. Chest. 2011;139:1025-1033. doi: 10.1378/chest.10-3011

29. O'Mara SK, Zborovskaya Y. End-of-life care in the Hispanic community. $J$ Hosp Palliat Nurs. 2016;18:53-59. doi: 10.1097/Njh.0000000000000210

30. Jennings LA, Zingmond D, Louie R, et al. Use of the physician orders for life-sustaining treatment among California nursing home residents. J Gen Intern Med. 2016;31:1119-1126. doi: 10.1007/ s11606-016-3728-9

31. Nicholas LH, Bynum JP, Iwashyna TJ, Weir DR, Langa KM. Advance directives and nursing home stays associated with less aggressive endof-life care for patients with severe dementia. Health Aff (Millwood). 2014;33:667-674. doi: 10.1377/hlthaff.2013.1258

32. Tellez-Giron P. Providing culturally sensitive end-of-life care for the Latino/a community. WMJ. 2007;106:402-406.

33. López L, Peralta CA, Lee A, Zeki Al Hazzouri A, Haan MN. Impact of acculturation on cardiovascular risk factors among elderly Mexican Americans. Ann Epidemiol. 2014;24:714-719. doi: 10.1016/j. annepidem.2014.07.011

34. Holmes JS, Driscoll AK, Heron M. Mortality among US-born and immigrant Hispanics in the US: effects of nativity, duration of residence, and age at immigration. Int J Public Health. 2015;60:609-617. doi: 10.1007/ s00038-015-0686-7

35. Wang SY, Aldridge MD, Gross CP, et al. End-of-life care intensity and hospice use: a regional-level analysis. Med Care. 2016;54:672-678. doi: 10.1097/MLR.0000000000000547

36. Mitchell SL. Advanced dementia. N Engl J Med. 2015;373:1276-1277. doi: 10.1056/NEJMc1509349 